\title{
Quest for the Fashion Market: Zero-Waste Skirt Design as a Solution toward Sustainable Pattern Making
}

\author{
Marzie Hatef Jalil ${ }^{1}$ \& Seyedeh Kobra Hosseini ${ }^{2}$ \\ ${ }^{1}$ PhD Candidate, Faculty of Applied and Creative Arts, Universiti Malaysia Sarawak, \\ Sarawak, Malaysia. Email: marzie.hatef@gmail.com. \\ ${ }^{2}$ Master in Textile and Fashion Design, Textile Engineering Department, Islamic Azad \\ University, Ghaemshahr, Iran.
}

\begin{abstract}
The present study shows the investigation of variables causing waste in the pattern making process through the examination of common methods of skirt design to ensure the optimal arrangement. Hence, efficiency is boosted during the pattern making phase by removing such variables. In this regard, it is proved the idea of making different skirts designs in the zero-waste approach without changing the skirt design structure. Moreover, the weight of pattern wastepaper is calculated after separating the garment parts from the main layout to evaluate the effect of creating a design regarding waste reduction. The study shows while maintaining the skirt design structure and using the standard pattern methods, appropriate changes are needed for a creative style of skirt design. The results can be used as a guideline to pattern makers and manufacturers for more objective selection and to support the development of zero-waste pattern making methods.
\end{abstract}

Keywords: Creative skirt design, fashion design, sustainable pattern making, wasteless waste reduction, zero-waste fashion.

\section{Introduction}

With the growth of population and the expansion of industry, environmental issues such as waste disposal, air pollution and water resources were more considered in society. The textile and clothing industry is responsible for $7 \%$ of carbon emissions in the atmosphere (Carrico \& Kim, 2014). The average fabric waste production in the cutting phase has been calculated at $15 \%$ (Carrico \& Kim, 2014). Moreover, the pattern design phase is the initial stage in the clothing production, where the layout of nesting process is one of the most challenges issues in the field of operations research (Tama \& Öndoğan, 2014). Owing to the different shapes of the garment pieces, unusable areas in the marker layout lead to making fabric waste. With accordance to the economic value of the materials, efforts are being made to provide the most suitable solution toward arrangement the nesting of garment pieces in the layout scale. Though attempts have been made to investigate ease levels, there are still no definitive guides as to what levels of ease should be incorporated into the pattern. The process of pattern construction has been the subject of much research, especially considering developing Computer-Aid Design (CAD) and measurement and nesting technologies, however, there are few studies provided insights into the provision of ease in the pattern making. Nevertheless, the total fabric waste has not been reduced to zero per cent. The main purpose of this step is to make maximum use of the fabric and minimize discarding fabric concerning zero-waste design in the clothing industry (Hatefjalil \&

(C) AesthetixMS 2020. This Open Access article is published under a Creative Commons Attribution Non-Commercial 4.0 International License (http://creativecommons.org/licenses/by-nc/4.0/), which permits non-commercial re-use, distribution, and reproduction in any medium, provided the original work is properly cited. For citation use the DOI. For commercial re-use, please contact editor@chitrolekha.com 
Shaharuddin, 2019). The limited information on zero-waste pattern design available to the researchers chiefly consists of the work of contemporary designers adopting a zero-waste approach including (Rissanen, 2008) and Holly (McQuillan, 2011), both of whom use the basic block as the foundation of their work. The purpose of the present article is to highlight using creativity in pattern making of the design phase as an approach in making the pattern with compelling design outcomes, and not only a methodology for sustainable fashion. It offers an insight for those practitioners unfamiliar with zero-waste pattern making design, to follow a different methodology to those already presented by the designers.

\section{Literature Review}

Regarding the pattern making process, the precise and guaranteed methods to find the best solution toward nesting the garment pieces were founded in 1950 (Armstrong \& Maruzzi, 2010). Due to the increasing degree in the complexity of calculations with increasing the size of the problem, it is not possible to find the optimal answer to these problems in a reasonable time. In order to overcome these problems, innovative methods were introduced such as Bottom left algorithms, belt algorithm, bottom left fill algorithms, the TOP piece selection (TOPS), and placement of the best fit (Rissanen, 2008). The complexity of the nesting procedure is closely related to the geometric shape of the garment pieces (Armstrong \& Maruzzi, 2010). In the nesting operation, the clothing industry is faced with the issue of the two-dimensional arrangement of block patterns. Despite all the proposed methods, efforts are needed to introduce more appropriate approaches. Designers and manufacturers have studied specific designs and pattern making methods concerning the reduction of fabric waste before the pattern cutting process (Armstrong \& Maruzzi, 2010). There is an idea to prevent waste during the pattern making process before final production. Creating a fit, which can be a design approach, is the basic idea to eliminate waste. This is not only a tool that each designer uses creativity in making clothes but also an initiative in the sewing process will be necessary (Carrico \& Kim, 2014). Creative pattern making and cutting are integral to the elevation and execution of contemporary fashion design (Townsend \& Mills, 2013). A creative pattern making methodology can be developed through imposing restrictions on traditional methods of working (Townsend \& Mills, 2013).

The zero-waste design was introduced in the 21st century. The concept of zero-waste enforces the idea of using all the fabric, either throughout a collection or within a single garment. Moreover, the term "zero-waste in the clothing industry" appeared in a study by Timo Rissanen (2008). Rissanen (2008) emphasized that development should be placed on the integration of pattern pieces, thus allowing the fabric to fall across and interact with the body, thereby accentuating the natural form. He introduced that three main ideas are inside the thinking of zero-waste designs (1) a completely fashion-based method, (2) seamless weaving method, and (3) a piece of cloth (A-POC). The first method is usually used to make clothes from knitted fabric. The pieces of clothing are woven separately and then sewn together. In the best shape, there are no cuts in the garment, so no yarn or fabric waste is produced (Rissanen, 2008; Rissanen \& McQuillan, 2016). The seamless weaving is a method in which the knitting machine weaves the final garment in three dimensions and the cutting and sewing are eliminated in the process. Knitted socks and gloves are examples of seamless textures. In the late 1990s, a Japanese designer, Izzy Miyaki, introduced a collection in the A-POC method. The customer buys a piece of fabric and cuts the final garment by following the connection among lines of the pieces.

Following this research, Rissanen (2008) introduced five zero-waste techniques in the apparel design. Moreover, McQuillan (2011) designed clothes without waste with using these 
techniques; (1) tessellation, (2) jigsaw, (3) embedded jigsaw, (4) multiple cloth approach and (5) minimal cut. The tessellation method includes a shape which is repeated exactly without space (McQuillan, 2011). In other words, a mosaic floor of the room is kind of a tessellation pattern. The design process of the jigsaw and the embedded jigsaw is implemented same with tessellation, however, the jigsaw method supports new techniques in producing multiple garments to increase efficiency and effectiveness (Saeidi \& Wimberley, 2018). In contrast with the tessellation method, these methods will allow the designers to use a variety of patterns and their creativity. The patterns are considered as puzzle parts which are placed in the appropriate way regarding the elimination of waste. These methods challenge all knowledge of designers (McQuillan, 2011; Saeidi \& Wimberley, 2018). In this case, the pattern designer should have a strong background in fabrics and their properties as well as pattern making techniques, clothing structure, fashion and process design (Saeidi \& Wimberley, 2018). For this article, researchers follow Rissanen's Jigsaw Puzzle method (Rissanen \& McQuillan, 2016), where all the collective pieces of the full garment pattern are engineered to fit the fabric width. Moreover, the multiple cloth approach is also a way to simultaneously design of two or more clothing patterns in one layout scale.

Some problems need to be overcome concerning zero-waste fashion techniques for the mass production of clothing (HatefJalil \& Shaharuddin, 2019). Research in the zero-waste pattern making has not been focused on grading and producing different sizes of clothing (HatefJalil \& Shaharuddin, 2019). Also, financial and environmental issues are not the only benefits of zerowaste clothing design. It might lead to that designers will introduce new styles of clothing in the fashion market, where these styles do not follow the usual trend. It is possible that the proposed design is inappropriate and will bring new samples to fashion trends which the idea conflicts with the existing clothing in the fashion market (Carrico \& Kim, 2014). Moreover, the zero-waste clothes on the fashion market are predominantly cut using the basic block and not as the whole garment structure. The block can be used as a guide, where the pattern maker fixes and controls areas where the rest of the design can be worked around and allowing for some experimentation (McQuillan, 2011). Alternatively, the block is utilized to create the main pattern, and the unused fabric is applied for waistbands and facings (Saeidi \& Wimberley, 2018).

\section{Methodology}

In the present study, three skirt designs called Straight, Pencil and Cloche (Flared) were considered for investigation (Armstrong \& Maruzzi, 2010). Then, the creative approach was applied to eliminate waste. First, the most appropriate method to guarantee the minimum waste was selected among three techniques of the flat pattern making such as the Metric, Muller and Basic Blocks pattern (Tama \& Öndoğan, 2014). Each technique develops a preference for construction methods based on pattern block shape (Gill \& Chadwick, 2009). Also, the darts and the skirt scale are mainly important issues in the skirt design. Basic Blocks pattern method for designing of the zero-waste straight skirt is more appropriate than the other two methods due to the design structure. Moreover, the combination of Muller and Basic Blocks pattern methods was used to design for other skirts. Primarily, the skirt's pattern was drawn in the best way and then the amount of wastepaper was obtained by cutting and calculation of waste pieces weight. In the next step, the process of the zero-waste design was done by (1) changing the arrangement of the garment pieces in the marker layout and (2) moving the inner lines, where both variables caused the waste. In this step, the same rules of zero-waste were used. If the waste is reduced to zero in several stages, the fabric and wastepaper are calculated for each stage and the waste reduction of each process will be evaluated. For this study, medium size (38) of the global standards was used 
for clothing measurement with a $94 \mathrm{~cm}$ hip circumference and a $68 \mathrm{~cm}$ waist circumference, and the desired skirt height.

\section{Results and Discussion}

There are different types of skirts that cover the lower part of the body, depending on the shape and size. Some factors such as designing the waistline, side seam, and the lower edge, play an important role in the skirt's formation. In general, the skirt is divided into three categories in terms of shape; straight, pencil and cloche. The rest of the types are derived in these domains.

\section{4-1 Straight skirt}

Straight skirt is one of the most important types of skirts, which its initial shape is like a cylinder and the side seams are parallel to the body's side. Finally, the skirt width is adjusted in the waist area by tying the darts coming from the front and back. The principle of drawing a straight skirt pattern is almost the same with Metric, Basic Blocks pattern and Muller methods. The only difference is the skirt width in the front and back and also how the darts are placed in the waistline. The design of a straight skirt is possible with all three methods but regarding minimization of waste and obtaining the simplest arrangement, the initial pattern of the straight skirt was drawn based on the Basic Blocks pattern method. Moreover, the proportions in the pattern are better observed in this pattern making method, which is expressed as an important principle in achieving zero-waste approach. Figure 1 (a) shows the archived pattern making flat of a straight skirt. After cutting the pattern pieces using the paper, the amount of waste paper was collected and weighed. The obtained weighing data is divided by the total weight of the pattern layout and expressed as a percentage to compare the amount of residual waste. In the straight skirt design, $15 \%$ of the total weight of the marked layout considered as waste. As shown in Figure 1 (a), a marked area in the pattern layout illustrates the amount of waste.
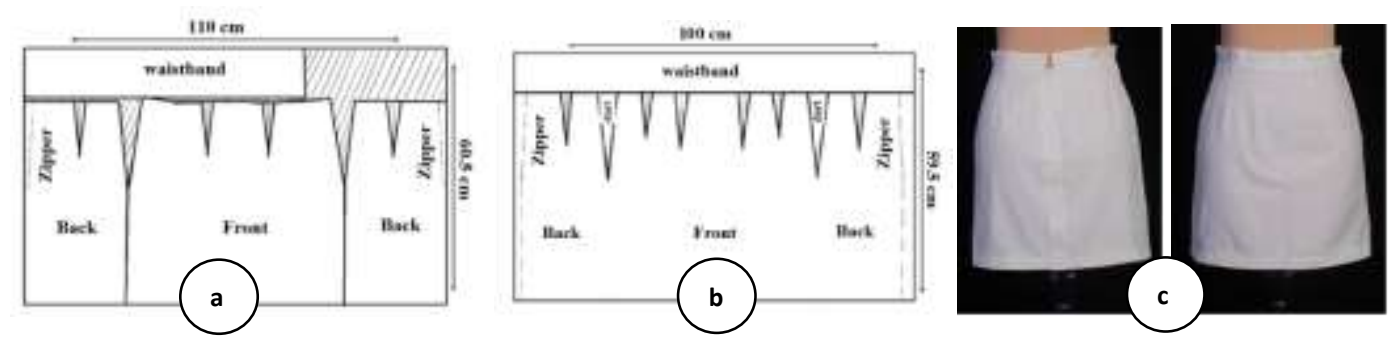

Figure 1: (a) Straight skirt pattern, (b) Zero-waste straight skirt, (c) Zero-waste straight skirt design

In Figure 1 (a), the small darts on the pattern layout are caused to make waste in the waistline area. Since the fabric width is usually more than the pattern layout width, which is calculated based on the hip circumference, hence it is possible to use the fabric width to extract the lumbar part of the skirt. This creativity leads to the reduction or elimination of waste. Because the initial pattern of the skirt is designed according to the standard method, it is possible to size and produces in mass scale. On the other hand, Figure 1 (b) shows the side waist area removed from the skirt. Also, the extra fabric of side waist was sewn in the waistline in the form of darts and the waist extra in the form of pleats regarding the removal of the waste. Hence, several skirt patterns can be placed in one marker layout at the same time and all fabric surface in the waistline area can be used. Thus, the fabric wastes are removed in case of mass production based on the zero-waste approach. Indeed, this type of layout maintains the classic style and is suitable for mass production of clothes. Therefore, the pattern designer will take action to remove the 
waste based on the skirt height, consumer size, skirt model, material, and fabric width, which all make waste in the clothing production. Moreover, Figure 1 (c) shows the straight skirt prototype created in the zero-waste design in front and back view.

\section{4-2 Pencil Skirt}

The initial shape of a pencil skirt is a polygon. The skirt waist should be exactly equal to the person's waist measurement by making pleats and darts. The skirt side is narrowed at the bottom edge. The pencil skirt is designed and modelled by Basic Blocks pattern and Muller methods. The only difference between these two methods is how to get the skirt tight. Indeed, the straight skirt pattern results in creating the pencil skirt pattern in the Basic Blocks method. Additionally, this skirt is drawn in two techniques in the Muller method. Rudimentary, in the first technique, the pencil skirt pattern is designed based on the straight skirt pattern and the skirt waist adjusted with pleats. Also, in the second technique, the skirt side is slightly narrowed down which is same as the structure of Basic Blocks pattern method (Armstrong \& Maruzzi, 2010).

Both pattern-making methods can be used for designing a zero-waste pencil skirt, however, Basic Blocks pattern method was used for this study. In this case, $10 \%$ of the total weight of the pattern layout is wasted. The marked areas as waste are shown in Figure 2 (a). Most fabric waste is produced due to the narrowing of the side seam. The re-pattern making of the pencil skirt was done to eliminate the waste using two solutions. Firstly, the curved side seam was neglected, and the three pieces of the skirt were continuously considered, and the pencil shape of the skirt was obtained by sewing darts on the waistline area (Figure 2 (b)). Secondly, the small darts were transferred to the large darts on the front, and the extra piece was sewn as a pleat. Moreover, Figure 2 (c) shows the pencil skirt prototype in the zero-waste design in front and side view.
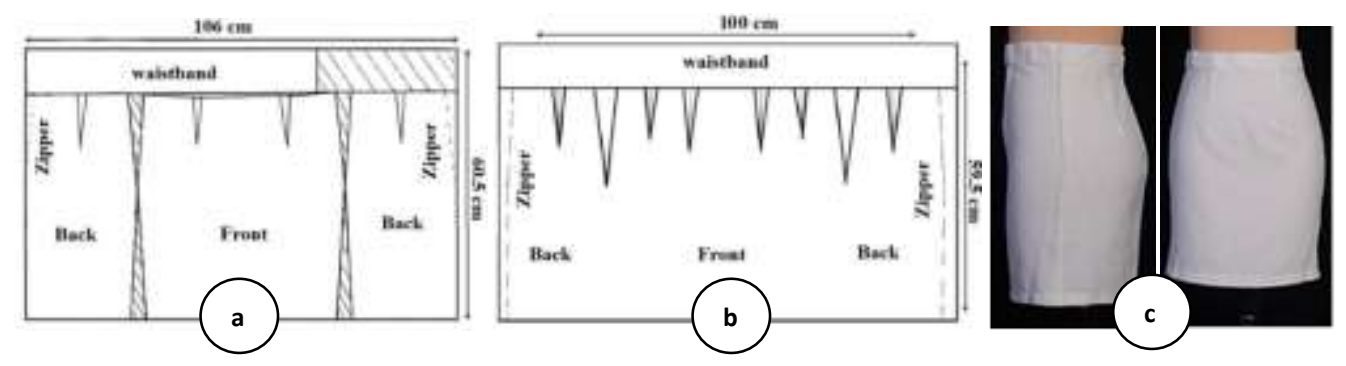

Figure 2: (a) Pencil skirt pattern, (b) Zero-waste pencil skirt, (c) Zero-waste pencil skirt design

As shown in Figure 3 (a), it is possible to design a pencil skirt through the base of the straight skirt based on the Muller method by creating pleats. Hence, the pattern layout was divided into two equal parts. Figure 3 (a) shows that the side seam was redefined, and the waistline was in one direction to remove the waste. Also, the pleats on the front and back were drawn in the same size and the extra fabrics were considered as a pleat. Moreover, Figure 3 (b) shows the pleated pencil skirt prototype in the zero-waste design in front and back view. 


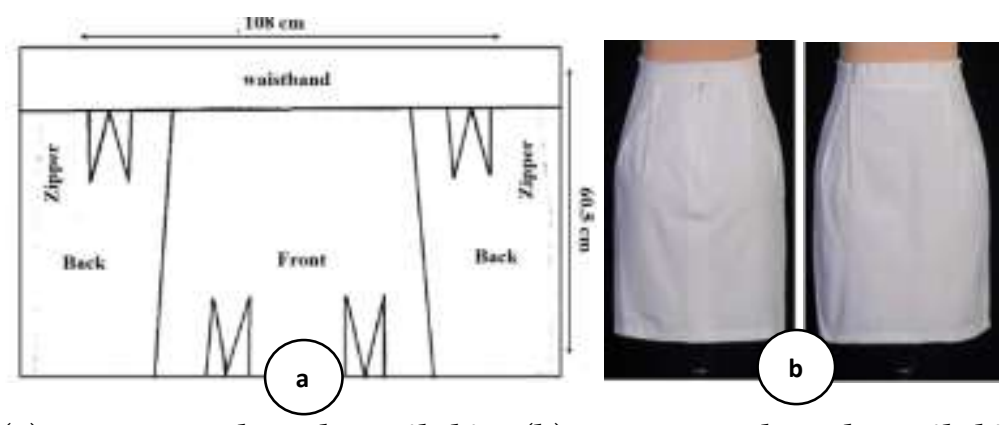

Figure 3: (a) Zero-waste pleated pencil skirt, (b) Zero-waste pleated pencil skirt design

\section{4-3 Cloche Skirt}

The initial shape of the cloche skirt is polygonal. The skirt waistline is tight and the looseness of the skirt at the bottom is caused by wrinkles. Moreover, the cloche skirt is divided into three categories.

A-line cloche. A-line cloche skirt has a zip seam on the back, and it is a fitted skirt in the waistline and starts to be wider. The cloche skirt has pleats and side seams in the Muller method. Also, the pleats can be closed or turned into a side seam (Armstrong \& Maruzzi, 2010). A-line cloche skirt is designed by two techniques. First, the pattern was arranged in the usual technique (the fabric width is $150 \mathrm{~cm}$ ). Since the pattern pieces are placed in one direction and next to each other, the pattern width will be more than the fabric width. Hence, this problem can be solved by a reduction of the skirt height to $49 \mathrm{~cm}$. In this case, $40.3 \%$ of the total weight of the pattern layout is wasted. Figure 4 (a) shows the marked areas in the layout as waste which is a considerable amount. Therefore, the pattern arrangement was changed due to a large amount of waste for the first time. As shown in Figure 4 (b), the amount of waste paper is reduced to $40.2 \%$ by raising two patterns on the back pieces of the A-line cloche skirt in the layout marker, but the layout length is increased from $80.5 \mathrm{~cm}$ to $90.5 \mathrm{~cm}$. It means there is no significant difference between first and second common techniques regarding making waste.

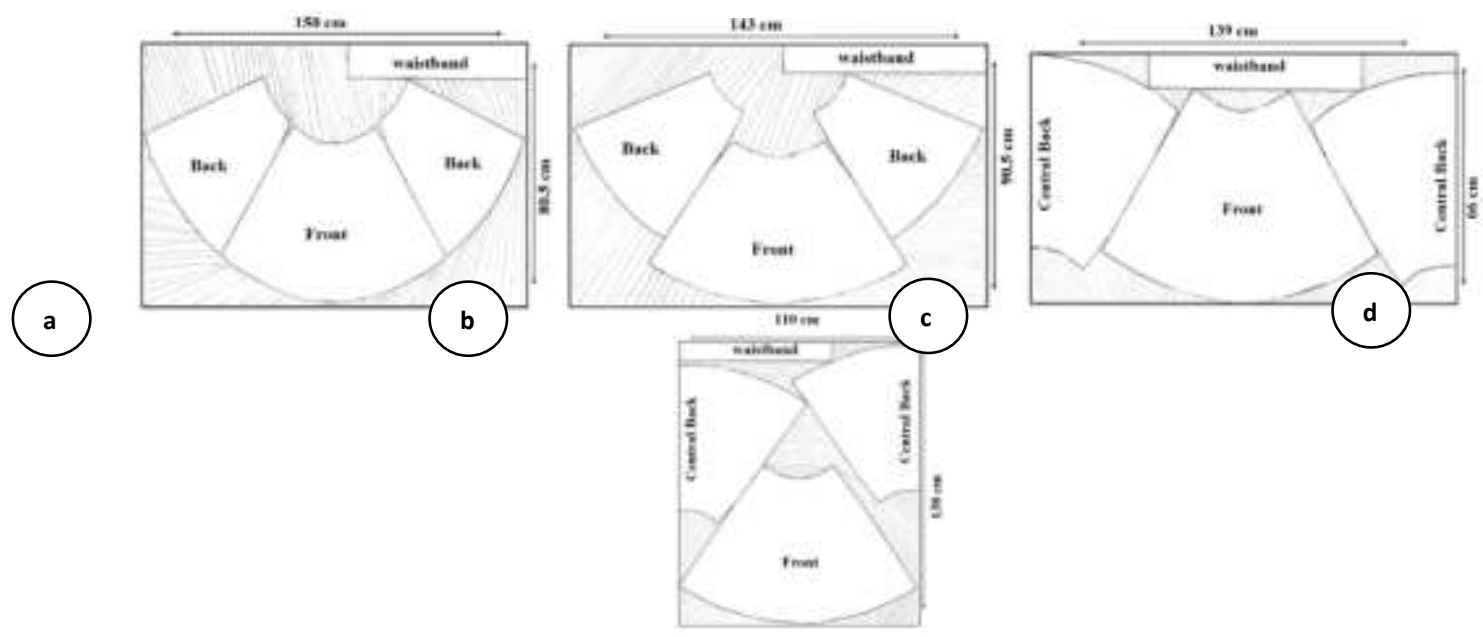

Figure 4: (a) A-line cloche skirt pattern (first technique), (b) A-line cloche skirt pattern (second technique), (c) A-line cloche skirt pattern (third technique), (d) A-line cloche skirt pattern with $70 \mathrm{~cm}$ height

Hence, creativity can help designers to resolve this problem. As shown in Figure 4 (c), the pattern was designed on the reverse side to further reduce waste in the third arrangement. Figure 
4 (c) shows that the marked areas in the pattern layout were greatly reduced and the amount of waste was decreased to $16.9 \%$. The length of the fabric used to produce the skirt would be increased by the changing of the skirt height. However, arranging the patterns of the cloche skirt with a $70 \mathrm{~cm}$ in height in the common technique leads to make $25 \%$ waste. The marked parts of the pattern layout are shown in Figure 4 (d) as a wasted area.

It is possible to remove waste in the production of these two skirts. The amount of waste was decreased by a slight reduction of skirt width created by rearranging the small patterns as shown in Figure 5 (a). The decorative crescent pieces were designed in such a way that the rest of the fabric was used to produce a decorative in the front area. The extra fabrics were extended from the back to the front in the form of the decorative pieces. Figure 5 (a) shows the layout of a skirt pattern with the $70 \mathrm{~cm}$ height, all parts were used in the skirt production with no waste. Moreover, Figure 5 (b) shows the A-line cloche skirt prototype in the zero-waste design in front and side view.
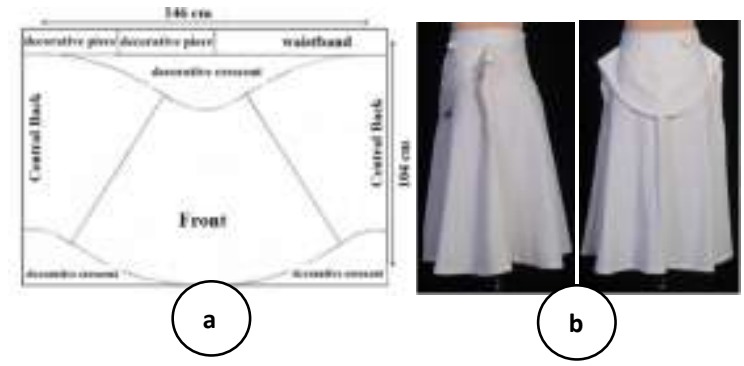

Figure 5: (a) Zero-waste A-line cloche skirt, (b) Zero-waste A-line cloche skirt design

Half Cloche Skirt. This type of skirt is tight at the waist and starts to be wider from the lower edge of hipline toward side seams. This skirt is designed by three methods: Basic Blocks pattern, Metric and Muller. All three methods can support the zero-waste design. In the present study, Basic Blocks pattern method was used to design for half cloche skirt with an overlapping hem on the front. As shown in Figure 6 (a), after arrangement the pieces, 33.3\% of the total weight of the pattern layout was wasted as a marked area. Hence, some parts in the marker layout were used as decorative pieces to remove the fabric waste (Figure 6 (b)). Figure 6 (c) shows the half cloche skirt prototype with following the zero-waste design in front, back and side view.

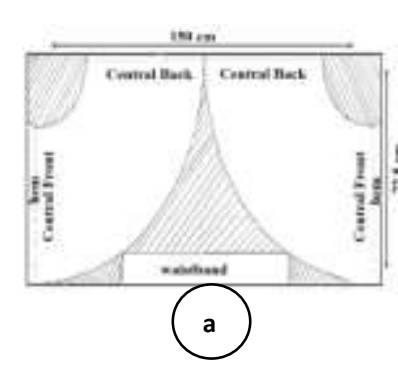

Figure 6: (a) Half cloche skirt pattern, (b) Zero-waste half cloche skirt design, (c) Zero-waste half cloche skirt design

Full Cloche Skirt. This type of skirt has many folds with a wide opening from the waist to the lower edge of the hip-high. The height of the conical folds usually extends to the waist area. It is only possible to make a pattern of the skirt based on the Muller and Basic Blocks methods. In 
the present study, Muller was accepted to design a full cloche skirt. As shown in Figure 7 (a), if the full cloche skirt is drawn based on the Muller method, $20 \%$ of the total weight of the pattern paper is wasted. Hence, none of the four main skirts in the lower edge should be cut into curves in the design of the full cloche skirt. Figure 7 (b) shows the square shape resulted in making a congressional shape after the sewing phase and the middle circle of the pattern layout, which is fabric waste, was used to sew a decorative pocket that is sewn to the side of the skirt. Figure 7 (c) shows the full cloche skirt prototype in the zero-waste design in front, back and side view.

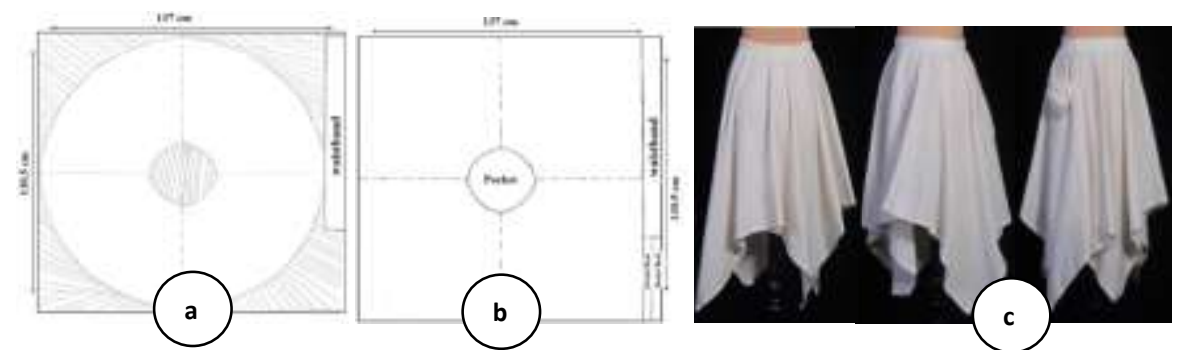

Figure 7: (a) Half cloche skirt pattern, (b) Zero-waste half cloche skirt design, (c) Zero-waste half cloche skirt design

Following this research, some of the skirt designs presented in fashion resources were selected for the idea generation. First, the skirt design was prepared as its common pattern making technique. Thereafter, the amount of wastepaper was calculated after extracting the pieces from the main layout. Then, the patterns were reviewed based on the designers' creativity and then, a new structure of the skirt was presented without waste. Hence, Figure 7 (a) shows a new design of the godet skirt with a crocheted hem.
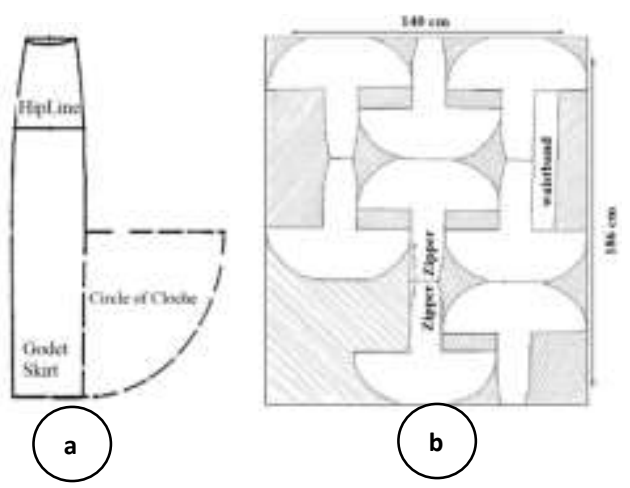

Figure 7: (a) Godet cloche skirt, (b) Godet skirt pattern design with 8 pieces

The basic pattern of the eight-godet skirt was drawn by the Basic Blocks pattern method. As shown in Figure 7 (b), $42 \%$ of the wastepaper was caused by arranging the pattern of this skirt is a common technique, where the radius of the cloche was $25 \mathrm{~cm}$. Hence, the variables caused the waste was discovered. The first variable was the opening of the cloche in the transverse part. So, the cloche width was reduced by $10 \mathrm{~cm}$ in width without reducing the height. Hence, the wasted paper was decreased to $25 \%$ (Figure 8 (a)).

Moreover, the second variable was that the length of the cloche piece was not equal with the skirt height. Hence, height was halved from the skirt waist and transferred to the waist-line part. Due to the incompatibility of the patterns, the sewing place was not added to the upper part of the godet. The waistline is diagonal; thus it was transferred to the extra crescent of the skirt leg. Also, the waist extra pieces were drawn as a pleat in the waist area. Therefore, the waist piece was 
drawn on the side and its curvature was designed through creating pleats. The crescent part of the skirt was used as a decorative part. The extra crescent was sewn to the sides as a decorative strap. The waistband was also considered as a belt inside the decorative strap (Figure 8 (b)). Moreover, Figure 8 (c) shows the godet skirt prototype in the zero-waste design in front, back and side view.
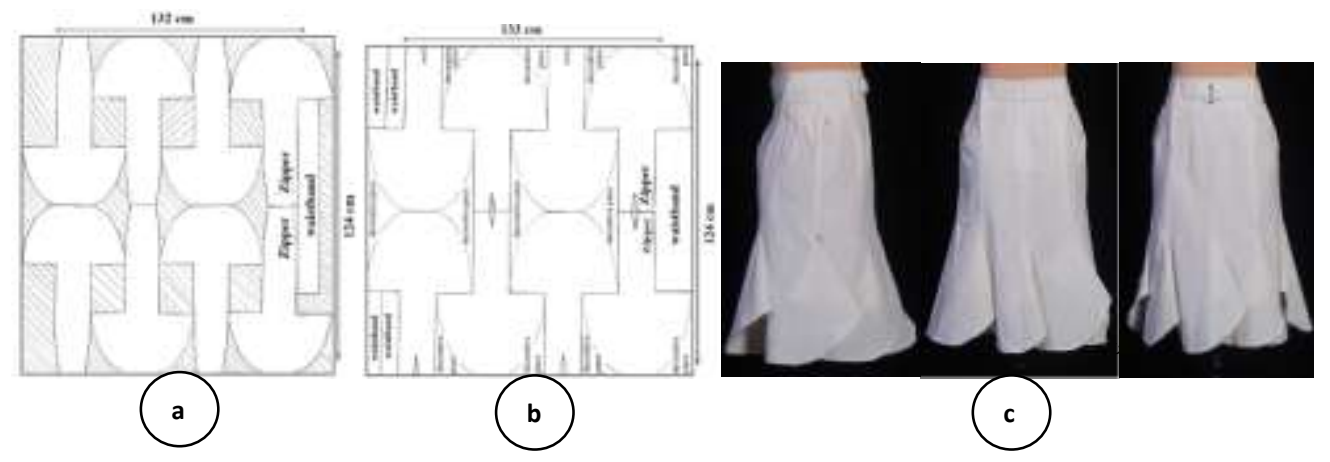

Figure 8: (a) Godet skirt pattern design (first factor), (b) Zero-waste godet skirt pattern design, (c) Zero-waste godet skirt design

Another eight-godet skirt was designed with a straight line on one side and a cloche part on the other side. In this idea, the skirt length was halved, and the cloche radius was half the skirt height. As shown in Figure 9 (a), the waste paper of this pattern is $11 \%$. According to the aims of this study, the excess seam at the edge of the crescent at the bottom was reduced regarding the zero-waste idea. As a view of creative design, it was formed by changing the lines and sewn together. The extra parts of the crescent at the bottom were sewn as pleats on both sides. Moreover, the extra waist parts were considered in the form as a waistband (Figure 9 (b)). Figure 9 (c) shows a creative design of the godet skirt in front, back and side view. Basic patterns, pattern layout and specific designs of clothing are originated from creative designers, where it can be an important inspiration source for design without or minimal waste.
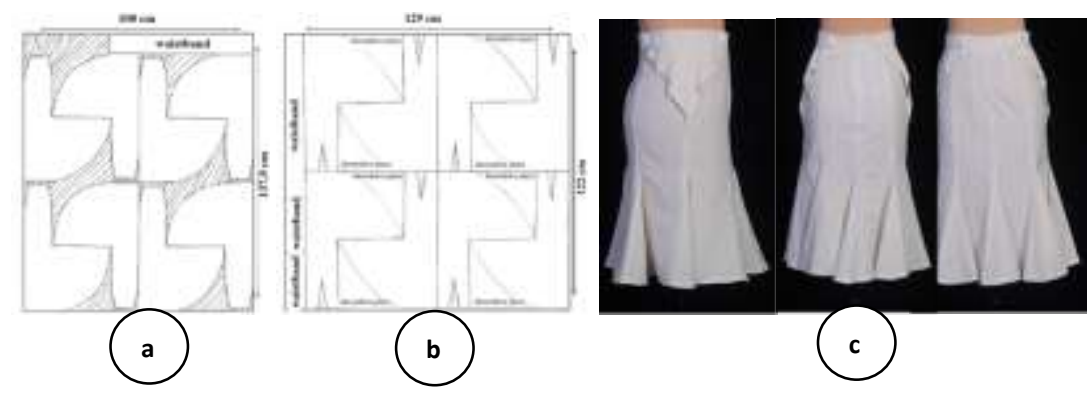

Figure 9: (a) Godet skirt pattern design (second factor), (b) Zero-waste godet skirt pattern design, (c) Zerowaste godet skirt design

\section{Conclusion and Recommendations}

The maximum usage of raw materials in the textile and clothing industry is one of the requirements for minimizing costs and helping to preserve the environment as well. The textile and clothing industry is a big polluter in the environment. A process such as disposal of fabric or clothing and other necessities in the garment production makes waste. A few types of research have been done studies to reduce the waste on the arrangement of markers or nesting process in the clothing production. Hence, each creative method makes some extent to optimize the marker 
layout and reduce fabric waste. However, the main issue has been to eliminate or reduce the waste after its production in the pattern making stage. Due to the use of sophisticated engineering algorithms, the proposed methods are incomprehensible to everyone, especially the pattern designer, who often lacks enough knowledge of zero-waste. Therefore, the elimination of waste in the previous methods was impossible and, also was not applicable on a mass-production scale.

On the other side, the lack of pattern sizing and the confrontation of new designs with the classic and conventional forms were big challenges in these techniques. Consequently, the present study attempted to eliminate some of these problems. The three types of skirts were accepted to be investigated as named Straight, Pencil and Cloche. The common pattern of these skirts was drawn in the medium size (38) and based on the Muller and Basic Block pattern making methods. The amount of pattern wastepaper was calculated in per cent. Then, the standard and suitable changes were recognized by the pattern designer based on creativity and using the rules of pattern drawing. Moreover, all designs followed that no part of the pattern layout remained as waste.

In the viewpoint of designers, many variables such as fabric width, fabric design, skirt height and skirt size are limitations during the design process toward zero-waste design. Findings showed that the pattern designer was successful in removing waste through redefining the pleats in terms of location and dimensions, as well as modifying the pattern layouts on the front and back. In this study, all eight different types of skirt patterns have been proven conventionally and creatively by removing the waste in new designs. One of the highlights of this research is that while eliminating waste in the pattern design phase, the overall shape of the skirts was not different from the normal form in the fashion market. It is possible to grade clothing to smaller and larger size based on the fabric width. Since there is no limitation for the designer's creativity. So, the initial patterns were designed based on the standard method and it is possible to produce different forms of the skirt on a mass-production scale.

All in all, ideas can be implemented in new designs toward elimination waste instead of being inspired by natural elements to design new clothes. Moreover, zero-waste pattern design process provides an excellent opportunity for a blended learning approach to make a creative design. Taking experiential knowledge and applying within a sustainable framework presents new looks for pattern makers/designers to solve existing problems, which leads to think and design holistically.

\section{References}

Armstrong, H. J., \& Maruzzi, V. J. (2010). Pattern making for fashion design: Prentice hall.

Carrico, M., \& Kim, V. (2014). Expanding zero-waste design practices: a discussion paper. International Journal of Fashion Design, Technology and Education, 7(1), 58-64.

Gill, S., \& Chadwick, N. (2009). Determination of ease allowances included in pattern construction methods. International Journal of Fashion Design, Technology and Education, 2(1), 23-31.

HatefJalil, M., \& Shaharuddin, S. S. (2019). Adopting C2CAD Model To Eco Capsule Wardrobe Design. International Journal of Scientific \& Technology Research (IJSTR), 8(12), 1224-1233. 
McQuillan, H. (2011). Zero-waste design practice: Strategies and risk-taking for garment design. Shaping sustainable fashion: Changing the way we make and use clothes, 83-97.

Rissanen, T. (2008). Creating fashion without the creation of fabric waste. Sustainable Fashion why Now? A conversation about issues, practices and possibilities.

Rissanen, T., \& McQuillan, H. (2016). Zero waste fashion design (Vol. 57): Bloomsbury Publishing.

Saeidi, E., \& Wimberley, V. S. (2018). Precious cut: exploring creative pattern cutting and draping for zerowaste design. International Journal of Fashion Design, Technology and Education, 11(2), 243-253.

Tama, D., \& Öndoğan, Z. (2014). Fitting evaluation of pattern making systems according to female body shapes. Fibres \& Textiles in Eastern Europe.

Townsend, K., \& Mills, F. (2013). Mastering zero: how the pursuit of less waste leads to more creative pattern cutting. International Journal of Fashion Design, Technology and Education, 6(2), 104-111. 